\title{
GALAXY EVOLUTION IN DISTANT CLUSTERS
}

\author{
B.M. POGGIANTI \\ Kapteyn Instituut \\ P.O. Box 800, 9700 Groningen, The Netherlands \\ AND \\ G. BARBARO \\ Dipartimento di Astronomia \\ vicolo dell'Osservatorio 5, 35122 Padova, Italy
}

A significant evolution has been detected in intermediate redshift clusters $(z<0.9)$, first by photometric studies ([1], [2]), which showed an excess of blue objects; subsequent spectroscopic studies revealed anomalies in most of the galaxies, mainly consisting of excessively strong Balmer lines. In order to explain the spectroscopic observations, bursts of star formation superimposed to the traditional scenario of galactic evolution are needed. The analysis of spectral lines and colours by means of an evolutionary synthesis model ([3]), including both the stellar contribution and the emission of the ionized gas, allows in most of the cases the determination of the time elapsed since the end of the burst and the fraction of galactic mass involved in it. In the clusters considered (AC103, AC114, AC118 at $\mathrm{z}=0.31$, [4]), the theoretical analysis demonstrates that the bursts affect substantial galactic mass fractions, typically $30 \%$ or more. The observations can be equally well reproduced by either elliptical+burst models or by spiral+burst models in which the star formation is truncated at the end of the burst. The analysis of an UV colour such as (1550-V) is proposed as a valid method to distinguish between the two cases for $\mathrm{H} \delta$ strong red galaxies.

\section{References}

1. Butcher H.R., Oemler A.Jr, 1978, ApJ 219, 18

2. Butcher H.R., Oemler A.Jr, 1984, ApJ 285, 426

3. Poggianti B.M., 1995, PhD Thesis, University of Padova

4. Couch W.J., Sharples R.M., 1987, MNRAS 229, 423 